\title{
Microinversor Tipo Flyback con Control Nolineal para Aplicaciones Fotovoltaicas de Baja Potencia
}

\author{
Rivera, Pablo ${ }^{1, * i D}$; Sotomayor, Nelson ${ }^{1}$ \\ ${ }^{1}$ Escuela Politécnica Nacional, Departamento de Automatización y Control Industrial, Quito, Ecuador
}

\begin{abstract}
Resumen. En este trabajo se presenta una solución sencilla para el diseño de un controlador de un conversor integrado al panel fotovoltaico basado en un inversor tipo Flyback de baja potencia con control nolineal de la corriente del primario con el propósito de controlar la potencia inyectada a la red eléctrica con factor de potencia unitario. La corriente de referencia es obtenida utilizando el algoritmo de Conductancia Incremental (IC) y en consecuencia obtener la potencia máxima del panel solar ante cualquier cambio de las condiciones atmosféricas. El controlador nolineal es diseñado para trabajar en el modo de conducción discontinua (DCM) y de esta manera facilitar el diseño si se compara con la complejidad de diseño que presenta este tipo de conversores cuando funciona en el modo de conducción continua (CCM) debido a que la función de transferencia de la corriente del inversor tipo Flyback presenta un cero en el semiplano derecho (RHP). Adicionalmente, se desarrolla el análisis de estabilidad utilizando el método de Lyapunov para demostrar la estabilidad y delimitación de las señales del sistema en lazo cerrado. Los resultados de la simulación validan el funcionamiento y robustez del controlador.
\end{abstract}

Palabras clave: Inversor, Flyback, Celda Fotovoltaica, Control Nolineal.

\section{Flyback Microinverter with Nonlinear Control for Low Power Photovoltaic Applications}

\begin{abstract}
This work presents a simple solution for the design of a controller of an integrated converter into the photovoltaic panel based on a low power Flyback type inverter with nonlinear control of the primary current to control the power injected into the grid with unit power factor. The reference current is obtained using the Incremental Conductance (IC) algorithm and consequently attain the maximum power of the solar panel regardless of the atmospheric conditions. The nonlinear controller is designed to work in discontinuous conduction mode (DCM) facilitating the design when compared to the design complexity of this type of converters when operating in continuous conduction mode (CCM) due to the current transfer function of the Flyback inverter has a right half-plane (RHP) zero. Additionally, the stability analysis is developed using the Lyapunov method to demonstrate the stability of the system and the boundedness of the closed-loop system signals. The simulation results validate the performance and robustness of the controller.
\end{abstract}

Keywords: Inverter, Flyback, Photovoltaic Cell, Nonlinear Control.

\section{INTRODUCCIÓN}

La energía fotovoltaica es una de las más importantes fuentes de energía renovable que aparece como solución al rápido crecimiento de la demanda de energía e incremento de la contaminación ambiental debido al uso de combustibles fósiles. Abbou et al. (2016).

La alta incursión de sistemas fotovoltaicos monofásicos de baja potencia a las redes de generación distribuida, ha centrado la atención de las investigaciones de sistemas fotovoltaicos con microinversores integrados a cada panel solar con la finalidad de suplir las pérdidas de potencia debido al efecto sombra o a las características distintas de las celdas fotovoltaicas. En la mayoría de las aplicaciones fotovoltaicas, los paneles solares son conectados en serie y/o en paralelo para alimentar un conversor central pero la confiabilidad del sistema disminuye en caso de falla del inversor central. Islam et al. (2015). Para obtener el máximo punto de operación se utilizan distintos algoritmos de seguimiento del punto de máxima potencia (MPPT), pero una pérdida considerable de energía puede producirse debido a la orientación variable de los paneles solares y al efecto de sombra (Romero et al., 2013).

Con el fin de solucionar los problemas de eficiencia debido al efecto sombra, en la literatura existen muchas investigaciones sobre métodos para obtener el punto de máxima potencia tales como en Ji et al. (2015) y MacAlpine et al. (2013). El uso de los conversores flyback conectados a un inversor para obtener corriente alterna desde un panel solar, ha llamado la atención 
de los investigadores por ser simple en estructura, bajo costo y alta eficiencia si se compara con otras topologías de inversores aislados ( $\mathrm{Li}$ et al., 2012). En la literatura científica existen estudios de módulos fotovoltaicos con inversor integrado, cuya topología tiene como enlace entre el módulo fotovoltaico y el inversor un conversor flyback. Las técnicas de control utilizadas son Control PI, Control Fuzzy, Control Optimo, Proporcional Resonante tal como en Li et al. (2012), Hamad et al. (2013), Fonkwe et al. (2014), Zhang et al. (2013) y Lee et al. (2016). Sin embargo, Lee et al. (2016) manifiesta que el diseño de los controladores es complejo debido a que, este tipo de configuraciones son muy inestables por la presencia del cero en el semiplano derecho (RHP) cuando funcionan en el modo de conducción continua (CCM) y no tienen un amplio rango de control. En tal sentido, Lee et al. (2016) presenta un control repetitivo (RC) tipo discreto con realimentación de la corriente de salida para asegurar la estabilidad del sistema en lazo cerrado funcionando en modo CCM. Pero de igual manera, el diseño del sistema es altamente complejo y el control repetitivo sufre de una inherente respuesta lenta (Dai et al., 2008).

En el presente trabajo, se propone el diseño de un sistema fotovoltaico conectado a la red eléctrica basado en micro inversor con conversor flyback y control nolineal tal como se indica en la Figura 1. A diferencia de otros trabajos como el presentado por Lee et al. (2016), se propone un método sencillo de control basado en control nolineal para seguir la trayectoria de corriente del primario actuando en el modo de conducción discontinua (DCM) cuya referencia de corriente, en este trabajo, es obtenida del algoritmo de Conductancia Incremental para seguir el Punto Máximo de Potencia (MPP) para cualquier valor de irradiación y/o de temperatura. Para inyectar potencia activa a la red cuya corriente esté en fase con el voltaje de la red eléctrica, se propone primeramente obtener en el primario del trasformador una onda de corriente DC pulsante cuya envolvente sinusoidal es similar a un sistema de rectificación. Para esto, la señal de control del transistor T1 será una onda sinusoidal PWM modulada con una señal sinusoidal rectificada (RSPWM) sincronizada a la frecuencia de la red eléctrica. La relación de trabajo es obtenida de un controlador nolineal. Los elementos de potencia de la etapa inversora (T2 y T3) son conmutados de manera alternada para obtener corriente alterna. La corriente alterna generada es inyectada a la red eléctrica la misma que está en fase con el voltaje de la red, garantizando de esta manera un factor de potencia unitario. La corriente alterna obtenida desde el inversor es conectada a la red eléctrica a través de un filtro de salida para garantizar una corriente sinusoidal con bajo contenido armónico.

\section{MODELO DEL INVERSOR FLYBACK}

El circuito equivalente del conversor flyback es similar al circuito del conversor buck-boost con la diferencia de que la inductancia fija es reemplazada por un transformador de doble devanado en el secundario. Cada secundario del trasformador es conectado a un inversor formado por diodos (D2, D3) y transistores (T2,T3) tal como se indica en la Figura 1, conmutados alternadamente cada transistor a una frecuencia de $120 \mathrm{~Hz}$ de tal manera de obtener una onda de alterna de frecuencia $f=60 \mathrm{~Hz}$ sincronizada con la red de alimentación.
Para el análisis se utiliza el modelo de un transformador ideal el que incluye la inductancia de magnetización $L_{m}$.

Además, se asume que la corriente del primario $i_{p r i}$ es modulada por una onda sinusoidal rectificada de tal manera

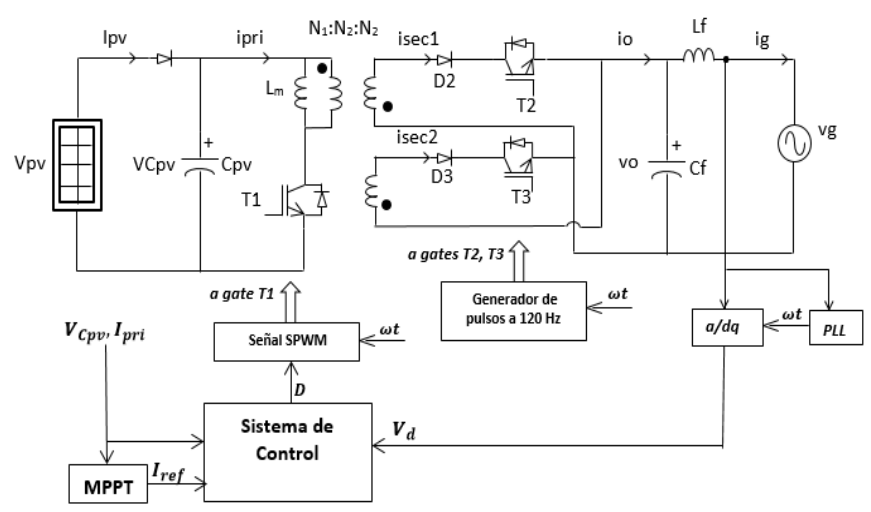

Figura 1. Circuito del inversor tipo Flyback conectado a la red eléctrica

que la corriente máxima del primario varíe sinusoidalmente. El transistor T1 es conmutado con la señal de control RSPWM a una frecuencia de conmutación $f_{s}$. La inductancia de magnetización es calculada de tal manera que el circuito funcione en el modo de conducción discontinua.

\subsection{Modo de Conducción Discontinua}

La Figura 2 indica una versión amplificada de la forma de onda de la corriente de magnetización cuya corriente del primario se da durante el tiempo Ton $_{n}$ de activado del transistor T1.

En el modo de conducción discontinua se cumple que:

$$
\operatorname{Ton}_{n}+\text { Tfall }_{n}<T s
$$

donde:

$\operatorname{Ton}_{n}$ es el tiempo de subida de la corriente del primario del transformador.

$\mathrm{Tfall}_{n}$ es el tiempo de bajada de la corriente de magnetización.

$T s$ es el tiempo de conmutación $=\frac{1}{f s}$

$$
\begin{gathered}
\text { Ton }_{n}=D_{n} T s \\
\text { Tfall }_{n}=d_{1 n} T s
\end{gathered}
$$

$D_{n}$ Relación de trabajo, donde "n" varía de 1 a $\frac{f s}{2 f}$ $d_{1 n}$ relación de trabajo de bajada de la corriente de magnetización. 


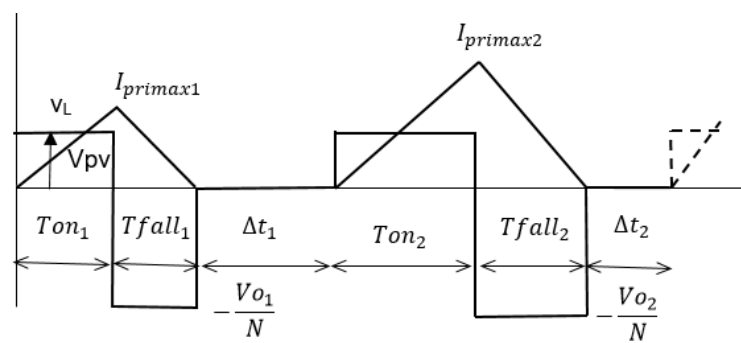

Figura 2. Forma de onda de voltaje y corriente de la inductancia de magnetización

En conducción discontinua, el tiempo de apagado $\operatorname{Tof} f_{n}$ está dado por la siguiente expresión:

$$
\operatorname{Toff}_{n}=\operatorname{Tfall}_{n}+\Delta t_{n}
$$

donde $\Delta t_{n}=d_{2 n} T s$;

$d_{2 n}$ relación de trabajo en corriente cero.

Asumiendo despreciable las pérdidas en el diodo anti-retorno, el valor máximo de la corriente del primario del transformador $I_{\text {primax }}$ varía sinusoidalmente de acuerdo a la siguiente relación tal como es presentada en Fonkwe et al. (2014):

$$
I_{\text {primax }}(t)=\frac{V p v \cdot D(t) \cdot T_{s}}{L m}
$$

donde:

$V p v$ es el voltaje de la celda fotovoltaica.

$D(t)$ es la relación de trabajo.

$L m$ es la inductancia de magnetización.

Durante el tiempo de activado Ton del transistor T1 la corriente media del primario $I_{p r i}(t)$ está dada por la siguiente relación:

$$
I_{\text {pri }}(t)=\frac{1}{2} D(t) I_{\text {primax }}(t)
$$

Reemplazando Ecuación (5) en Ecuación (6) se obtiene lo siguiente:

$$
I_{p r i}(t)=\frac{1}{2} \frac{V p v \cdot D^{2}(t) \cdot T_{s}}{L m}
$$

Asumiendo despreciables las pérdidas en el inversor y en el filtro de salida $L_{f}$, la potencia generada por la celda fotovoltaica Pin es igual a la potencia de salida Po (Le et al., 2016).

$$
V p v \cdot I p v=V g_{r m s} I g_{r m s}
$$

$V p v$ es el voltaje medio de la celda fotovoltaica $I p v$ es la corriente media de la celda fotovoltaica. $V g_{r m s}$ es el valor $r m s$ del voltaje de la red $I g_{r m s}$ es el valor $r m s$ de la corriente de la red.

Utilizando el principio de balance de potencia y asumiendo el factor de potencia unitario en el punto de acoplamiento común, el valor de la potencia instantánea está dado por la siguiente relación:

$$
V p v \cdot I_{p r i}(t)=2 V g_{r m s} I g_{r m s} \cdot \sin ^{2} w t
$$

donde $w$ es la frecuencia angular igual a $2 \pi f$.

Reemplazando las Ecuaciones (7) y (8) en la Ecuación (9) y luego de algunas operaciones matemáticas se obtiene el valor de la relación de trabajo en el modo de conducción discontinua:

$$
D(t)=2 \sqrt{\frac{\operatorname{Ipv\cdot Lm\cdot fs}}{V p v}}|\sin w t|
$$

Para determinar el valor crítico de la inductancia de magnetización, primeramente, se considera que el sistema funciona en conducción continua. Entonces, asumiendo funcionamiento en estado estable e igualando a cero la integral del voltaje en la inductancia de magnetización en el periodo $T s$ se obtiene la siguiente relación, (Le et al., 2016):

$$
D(t)=\frac{\sqrt{2} V g_{r m s} \cdot|\sin w t|}{N V p v+\sqrt{2} V g_{r m s} \cdot|\sin w t|}
$$

Donde $\mathrm{N}$ es la relación de transformación; $N=\frac{N 2}{N 1}, \quad \mathrm{~N}>1$ El valor crítico de la inductancia de magnetización se calcula considerando la condición más crítica; esto es, el valor máximo de la relación de trabajo en el límite de conducción continua y discontinua se determina en el valor pico del voltaje de la red es decir cuando sinwt $=1$. Luego, igualando las Ecuaciones (10) y (11) y después de algunas operaciones matemáticas se obtiene la expresión para determinar el valor crítico de la inductancia de magnetización $L m_{\text {critico }}$.

$$
L m_{\text {critico }}=\frac{V g_{r m s}^{2} \cdot V p v}{2 . I p v \cdot f s \cdot\left(N \cdot V p v+\sqrt{2} V g_{r m s}\right)^{2}}
$$

\section{DISEÑO DEL SISTEMA DE CONTROL}

El controlador no lineal asegura que el valor medio de la corriente del primario $I_{\text {pri }}(t)$ siga la referencia de corriente $I_{r e f}(t)$ obtenida del algoritmo seguidor del punto de máxima potencia (MPPT). El propósito del esquema de control es diseñar la relación de trabajo $D(t)$ de tal manera que $I_{\text {pri }}(t) \rightarrow I_{\text {ref }}(t)$ conforme $t \rightarrow \infty$ y de esta manera controlar la potencia activa entregada a la red eléctrica.

Para el diseño del controlador se asume lo siguiente:

Asunción 1. $R_{s}, C_{p v}, L_{m}, N$ son parámetros conocidos del sistema.

Asunción 2. El voltaje de la red eléctrica es simétrico y sinusoidal entonces la componente en eje directo $V d=$ $\sqrt{2} V g_{r m s}$ y la componente en eje en cuadratura $V q$ es cero. Asunción 3. El voltaje $V_{C p v}$ y corriente $I_{p r i}$ de la celda fotovoltaica son medibles.

Asunción 4. La corriente $I_{p r i}$ es delimitada conociendo que el voltaje $V_{C p v}$ es delimitado.

Asunción 5. La referencia de corriente $I_{\text {ref }}$ es delimitada y suficientemente diferenciable. 


\subsection{Modelo del sistema}

El circuito equivalente del sistema sin tomar en cuenta el efecto del filtro de salida es indicado en la Figura 3. Considerando que la resistencia Rs representa la resistencia serie interna del diodo anti-retorno conectado entre el capacitor de filtro $C_{p v}$ y la celda fotovoltaica, y que la corriente $i_{L m}$ en la inductancia de magnetización $L_{m}$ es igual a la corriente del primario $i_{\text {pri }}$ durante el tiempo de activado del transistor T1, las ecuaciones del modelo continuo son indicadas a continuación (Fonkwe et al., 2014).

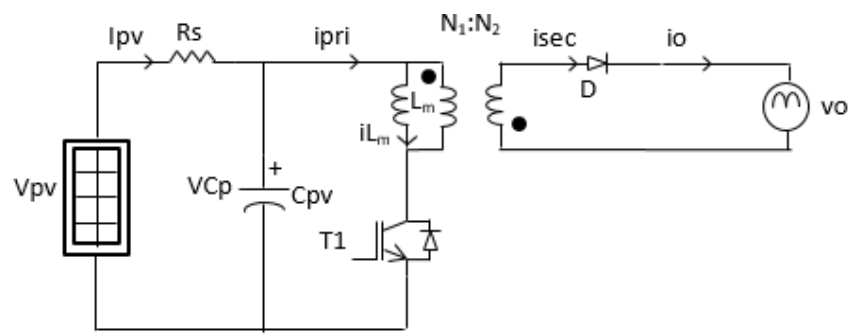

Figura 3. Modelo del inversor tipo Flyback

$$
\begin{gathered}
\dot{V}_{C p v}=-\frac{V_{C p v}}{R_{s} C_{p v}}-\frac{D}{C_{p v}} I_{p r i}+\frac{V p v}{R_{s} C_{p v}} \\
\dot{I}_{p r i}=\frac{D \cdot V_{C p v}}{L_{m}}-\frac{(1-D)}{N \cdot L_{m}} V_{o}
\end{gathered}
$$

Para conseguir la corriente deseada y de esta manera controlar la potencia activa abastecida a la red eléctrica, la señal de error $e_{1}(t)$ y su derivada son definidas como:

$$
\begin{aligned}
& e_{1} \triangleq I_{p r i}-I_{r e f} \\
& \dot{e}_{1} \triangleq \dot{I}_{p r i}-\dot{I}_{r e f}
\end{aligned}
$$

donde $e_{1}(t) \in \mathbb{R}$.

El sistema en lazo abierto es desarrollado reemplazando la ecuación (14) en (16) como sigue:

$$
L_{m} \dot{e}_{1}=D V_{C p v}-\frac{(1-D)}{N} V_{0}-L_{m} \dot{I}_{r e f}
$$

Para asegurar que la señal de error $e_{1}(t) \rightarrow 0$ conforme $t \rightarrow \infty$, la señal en lazo cerrado del error se escoge como:

$$
L_{m} \dot{e}_{1}=-K_{1} e_{1}
$$

donde $K_{1} \in \mathbb{R}^{+}$y representa la ganancia de control.

Substituyendo (18) en (17), luego de algunas operaciones matemáticas y considerando que $V_{0}=\left|V_{g}\right|$ se obtiene la expresión que determina la señal de la relación de trabajo $D$.

$$
D=\left(-K_{1} e_{1}+\frac{\left|V_{g}\right|}{N}+L_{m} \dot{I}_{r e f}\right) \frac{1}{\left(V_{C p v}+\frac{\left|V_{g}\right|}{N}\right)}
$$

donde $\left|V_{g}\right|$ es valor pico de la señal rectificada del voltaje de la red.

\subsection{Análisis de la estabilidad}

Se escoge una función de Lyapunov no-negativa como sigue:

$$
V_{1} \triangleq \frac{1}{2} L_{m} e_{1}^{2}
$$

La derivada de la Ecuación (20) es:

$$
\dot{V}_{1}=e_{1} L_{m} \dot{e}_{1}
$$

Para garantizar que la señal de error $e_{1}(t) \rightarrow 0$ conforme $t \rightarrow$ $\infty$, la señal en lazo cerrado del error es definida por la Ecuación (18). Luego, reemplazando (18) en (21) se obtiene la siguiente expresión:

$$
\dot{V}_{1}=-K_{1} e_{1}^{2}
$$

Puesto que la ganancia de control $K_{1} \in \mathbb{R}^{+}$es claro evidenciar que (22) es una función negativa definida. De Ecuación (22), $\dot{V}_{1}$ puede ser delimitada por el límite superior de la siguiente manera:

$$
\dot{V}_{1} \leq-K_{1} e_{1}^{2}
$$

De Ecuaciones (20) y (22) la señal de error $e_{1}$ es delimitada. De asunción 4 y conociendo que la señal de error $e_{1}$ es delimitado, de la ecuación (15) se concluye que $I_{\text {ref }}$ es delimitada.

Tomando en cuenta la asunción 1,2 y 5 conjuntamente con lo delimitaciones demostradas anteriormente, de la Ecuación (19) se puede determinar que $\mathrm{D}$ es delimitado. De lo demostrado arriba y la Ecuación (14) $\dot{I}_{p r i}$ es circunscrita. Entonces, conociendo que $\dot{I}_{\text {ref }}$ es delimitada de acuerdo a asunción 5 y tomando en cuenta la Ecuación (16), se puede determinar que $\dot{e}_{1}$ es delimitada. Finalmente, utilizando el teorema de estabilidad exponencial, propuesto por Khalil (2015), se puede demostrar que el origen; $e_{1}(t)=(0)$, es globalmente estable de manera exponencial.

\section{RESULTADOS DE LA SIMULACIÓN}

El paquete de simulación Matlab/Simulink con Simscape Power System se utilizó para modelar la dinámica del circuito del sistema fotovoltaico el que incluye el controlador nolinear. La simulación numérica fue desarrollada para validar el diseño del sistema de control. Los parámetros del sistema fotovoltaico son indicados en la Tabla 1, en donde el valor del capacitor del filtro $C_{p v}$ fue calculado de acuerdo a Hasan et al. (2017). El valor de la constante $K_{1}$ del controlador fue determinado por el método de ensayo y error hasta que la señal de error $e_{1}(t)$ en estado estable sea cero. Los valores de $C_{f} \mathrm{y}$ $L_{f}$ fueron determinados para eliminar el rizado de las componentes de la frecuencia $f_{s}$ de la corriente de salida de acuerdo a Macedo et al. (2016) y El Iysaouy et al. (2018).

\subsection{Cálculo de la Inductancia Crítica}

Para el cálculo de la inductancia crítica se consideran los parámetros de voltaje y corriente de la celda fotovoltaica 
indicados en la Tabla 1 para obtener la potencia máxima a una irradiación de $1000 \mathrm{~W} / \mathrm{m}^{2}$.

De Ecuación (12), considerando la relación de transformación $\mathrm{N}=10$ para obtener el voltaje pico de la red eléctrica igual a $\sqrt{2} V_{g}$ y una frecuencia de conmutación $f_{s}=24 \mathrm{KHz}$, se obtiene lo siguiente:

$$
L m_{\text {critico }}=5,56 \times 10^{-6}
$$

Tabla 1. Parámetros del Sistema Fotovoltaico

\begin{tabular}{cccc}
\hline \multicolumn{4}{c}{ MPP de la Celda a $25^{0} \mathrm{C}$} \\
\hline $\begin{array}{c}\text { Irradiancia } \\
\left(\mathbf{W} / \mathbf{m}^{\mathbf{2}}\right)\end{array}$ & $\begin{array}{c}\text { Corriente } \\
\text { Ipv }(\mathbf{A})\end{array}$ & $\begin{array}{c}\text { Voltaje } \\
\text { Vpv }(\mathbf{V})\end{array}$ & $\begin{array}{c}\text { Potencia } \\
\mathbf{P}(\mathbf{W})\end{array}$ \\
\hline 600 & 4,79 & 18,11 & 86,75 \\
800 & 6,37 & 17,89 & 113,96 \\
1000 & 7,94 & 17,6 & 139,9 \\
\hline Parámetro & Valor & Parámetro & Valor \\
\hline$C_{p v}$ & $12 \mathrm{mF}$ & $N$ & 10 \\
$C_{f}$ & $2,2 u F$ & $f$ & $60 \mathrm{~Hz}$ \\
$L_{f}$ & $10 \mathrm{mH}$ & $V_{g}$ & $120 \mathrm{Vrms}$ \\
$L_{m}$ & $0,004 m \mathrm{H}$ & $K_{1}$ & 2,7 \\
\hline \multicolumn{4}{c}{$f_{s}=24 \mathrm{KHz}$}
\end{tabular}

Para garantizar conducción discontinua, la inductancia para conducción discontinua $\mathrm{Lm}_{D C M}$ debe ser menor que $L m_{\text {critico }}$, luego se asume que:

$$
\begin{gathered}
L m_{D C M}=80 \% L m_{\text {critico }} \\
L m_{D C M} \approx 4,4 * 10^{-6}
\end{gathered}
$$

Con este valor de inductancia, se verifica si las relaciones de trabajo pico, calculadas utilizando la Ecuación (10), coinciden con los valores obtenidos de la simulación aplicando el control no-lineal para distintos valores de irradiación.

$$
\begin{gathered}
D(t)_{1000 \mathrm{~W} / \mathrm{m} 2}=2 \sqrt{\frac{7,94 * 0,004 * 10^{-3} * 24 * 10^{3}}{17,6}} \mid \text { sinwt } \mid \\
D(t)_{1000 \mathrm{~W} / \mathrm{m} 2}=0,41|\sin w t| \\
D(t)_{800 \mathrm{~W} / \mathrm{m} 2}=0,36 \mid \text { sinw } t \mid \\
D(t)_{600 \mathrm{~W} / \mathrm{m} 2}=0,31 \mid \text { sinw } t \mid
\end{gathered}
$$

La Figura 4 indica la relación de trabajo $\mathrm{D}(\mathrm{t})$ obtenida del controlador multiplicada por una señal unitaria sinusoidal rectificada para valores de irradiación que varían de $600 \mathrm{~W} / \mathrm{m}^{2}$ a $800 \mathrm{~W} / \mathrm{m}^{2}$, a $1000 \mathrm{~W} / \mathrm{m}^{2}$ y finalmente a $800 \mathrm{~W} / \mathrm{m}^{2}$ en tiempos $\mathrm{t}=0,0.4,0.8,1.2$ segundos. Como se puede ver en esta figura, los valores pico obtenidos del controlador son similares a los determinados analíticamente y la relación de trabajo varía de acuerdo a $|\sin w t|$.

La Figura 5 indica las formas de onda de la corriente del primario $i_{p r i}$ y las corrientes en los secundarios del trasformador $i_{\text {sec } 1}$ y $i_{\sec 2}$. Como se puede ver en esta figura, la corriente del primario tiene una envolvente sinusoidal rectificada con un pico de 75,4 A cuando la irradiancia es de $1000 \mathrm{~W} / \mathrm{m}^{2}$. De igual manera, las corrientes del secundario tienen un pico de 7,53 A corroborando la relación de transformación de $N=10$ considerada en este diseño. Es importante anotar que la desventaja del alto valor de la corriente pico cuando se trabaja en el modo de conducción discontinua es compensada por la facilidad de diseño del controlador no-lineal propuesto y la calidad de la energía obtenida.

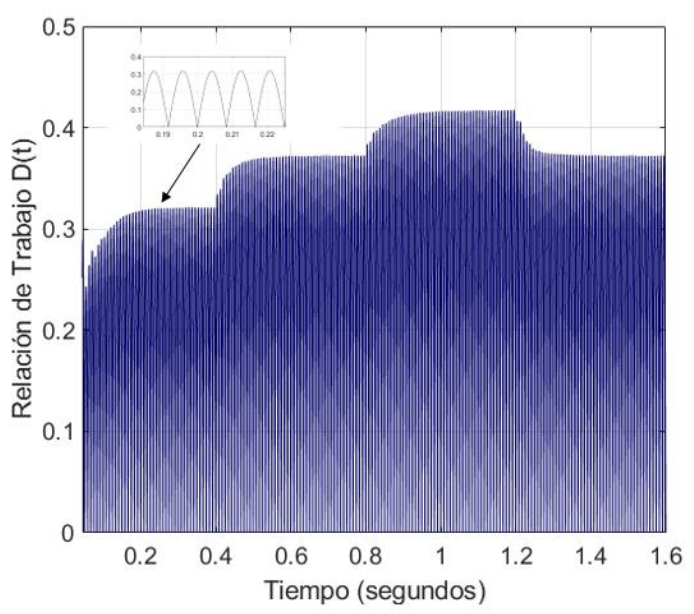

Figura 4. Forma de onda de la relación de trabajo $D(t)$
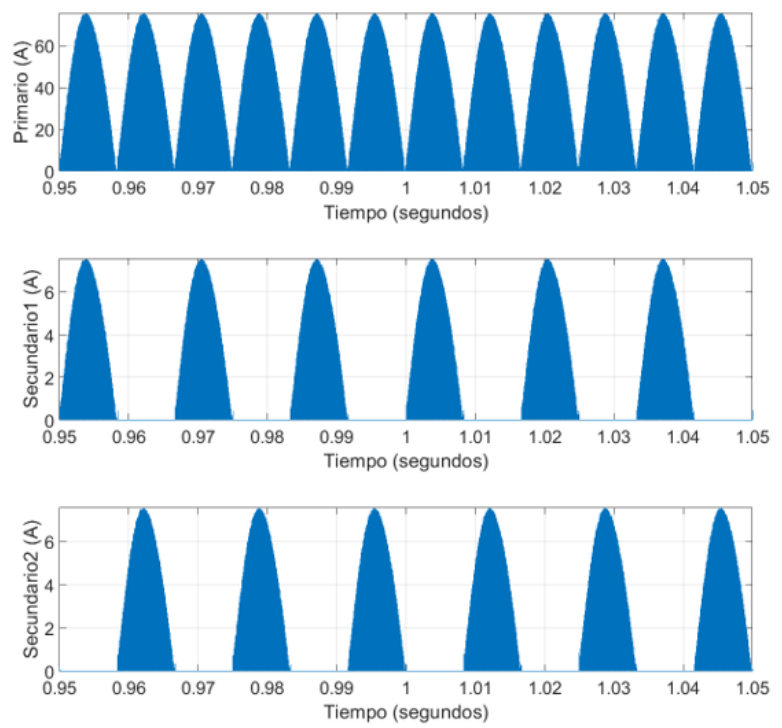

Figura 5. Formas de onda de la corriente del primario $i_{\text {pri }}$ y las corrientes del secundario $i_{\text {sec } 1}$ y $i_{\text {sec } 2}$ a una irradiancia de $1000 \mathrm{~W} / \mathrm{m}^{2}$

\subsection{Cambio de la Irradiancia}

Para verificar el correcto funcionamiento del controlador propuesto ante cambios bruscos de las condiciones atmosféricas, la irradiancia fue cambiada en pasos desde $600 \mathrm{~W} / \mathrm{m}^{2}$ a $800 \mathrm{~W} / \mathrm{m}^{2}$ a $1000 \mathrm{~W} / \mathrm{m}^{2}$ y luego una reducción a $800 \mathrm{~W} / \mathrm{m}^{2}$ en tiempos $\mathrm{t}=0,0.4,0.8,1.2$ segundos respectivamente manteniendo la temperatura en $25^{\circ} \mathrm{C}$. $\mathrm{La}$ Figura 6 indica la corriente de referencia $I_{r e f}$, obtenida del algoritmo Conductancia Incremental (IC); algoritmo adoptado en este trabajo debido a sus aplicaciones y buen desenvolvimiento en el seguimiento del MPP tal como manifiesta Ezinwanne et al. (2016), y el valor medio de la corriente del primario $I_{\text {pri }}$. 


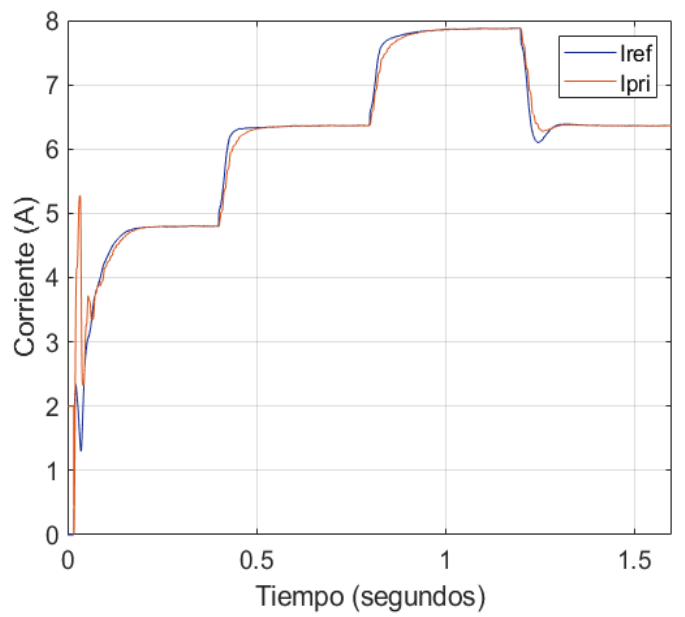

Figura 6. Formas de onda de la corriente de referencia Iref y el valor medio de la corriente del primario $I_{p r i}$

Como se puede ver en esta figura, la corriente $I_{p r i}$ sigue la referencia de corriente $I_{\text {ref }}$. Además, se puede ver en esta figura que en estado estable los valores medios de la corriente son aproximadamente iguales a los especificados por el fabricante del panel fotovoltaico para irradiancias de $600 \mathrm{~W} /$ $m^{2}, 800 \mathrm{~W} / \mathrm{m}^{2}$ y $1000 \mathrm{~W} / \mathrm{m}^{2}$, validando de esta manera el uso del algoritmo IC para obtener una corriente de referencia al cual se obtiene la máxima potencia y el algoritmo de control propuesto.

La potencia de salida $P_{p v}$ obtenida del sistema fotovoltaico y la potencial máxima ideal es indicado en la Figura 7. Se puede ver en esta figura que la Potencia $P_{p v}$ tiene una pequeña desviación de aproximadamente $2.2 \mathrm{~W}$ dando una eficiencia de aproximadamente $99.1 \%$ tomando como base la Potencia máxima ideal. Porcentaje aceptable al utilizar el algoritmo de Conductancia Incremental tal como el presentado por Kareim et al. (2013).

Para ver la respuesta de la corriente inyectada a la red ante cambios bruscos de la irradiación, para efectos de visualización y análisis, se toma el intervalo de tiempo entre 1.1 seg. y 1.3 seg. tal como se indica en la Figura 8. De igual manera, para efectos de visualización la forma de onda de corriente fue escala en 10 veces. La Figura 8 indica el voltaje y la corriente inyectada a la red cuando la irradiancia cambia de $1000 \mathrm{~W} / \mathrm{m}^{2}$ a $800 \mathrm{~W} / \mathrm{m}^{2}$ en el tiempo $\mathrm{t}=1.2$ segundos. Como se puede ver en la figura, cuando la irradiancia disminuye de $1000 \mathrm{~W} / \mathrm{m}^{2}$ a $800 \mathrm{~W} / \mathrm{m}^{2}$, la corriente disminuye de 16 Apico a 13.2 Apico (valores escalados). Además, se puede ver que la corriente inyectada a la red es sinusoidal y en fase con el voltaje de la red con un factor de potencia aproximado a la unidad inclusive en los puntos de cambios de la irradiancia, validando de esta manera la efectividad y rigidez del sistema fotovoltaico propuesto con control nolineal de la corriente del primario.

La Figura 9 indica la distorsión armónica total (TDH) para una irradiancia de $800 \mathrm{~W} / \mathrm{m}^{2}$. Se puede ver que el THD es de aproximadamente del $4.19 \%$ lo cual cumple con los límites especificados en las normas IEEE-519.

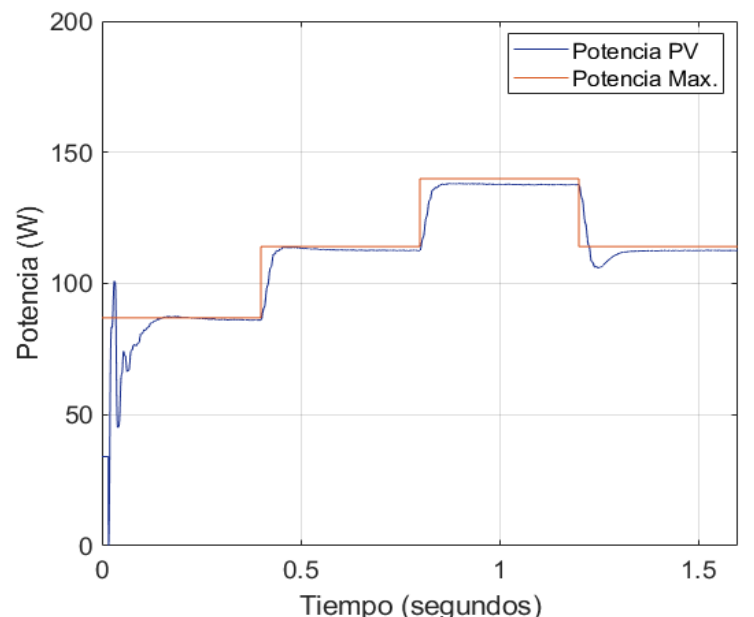

Figura 7. Potencia de salida de la celda fotovoltaica y Potencia máxima ideal

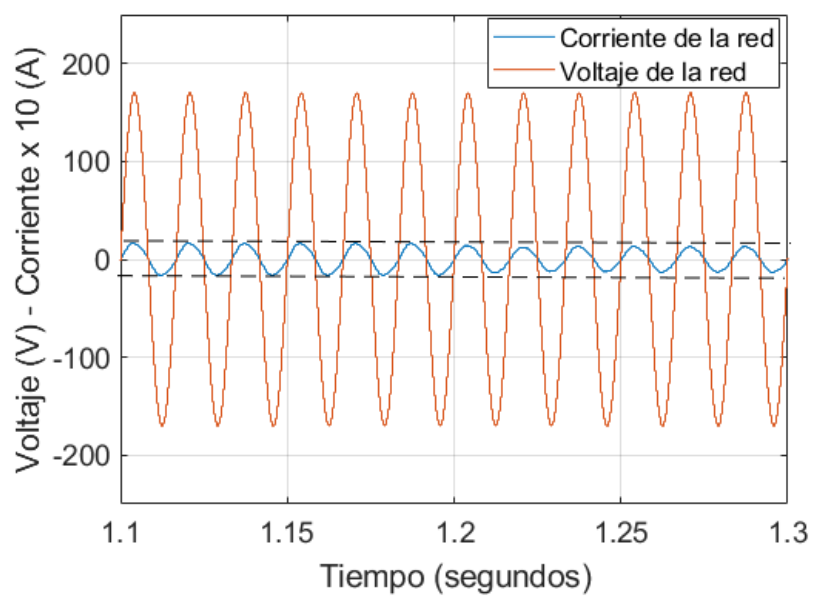

Figura 8. Voltaje y corriente de la red ante un cambio brusco de la irradiación al tiempo $\mathrm{t}=1.2$ segundos

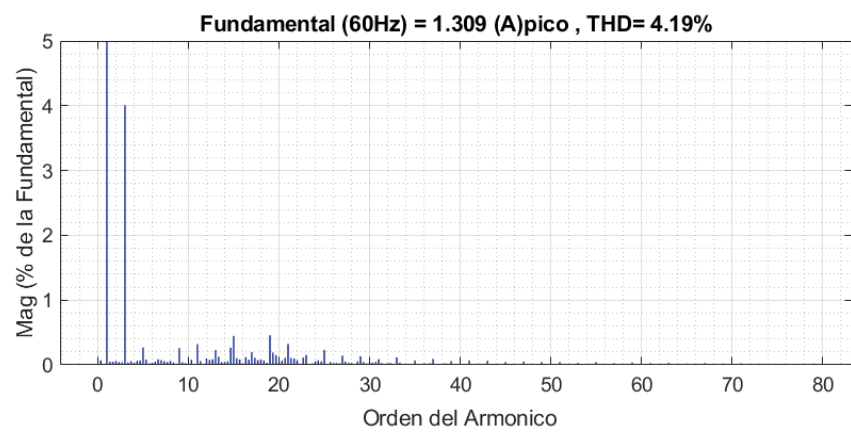

Figura 9. Distorsión armónica total de la corriente de la red a $800 \mathrm{~W} / \mathrm{m}^{2}$

\section{CONCLUSIONES}

Se demostró que el control nolineal propuesto fue efectivo en seguir la referencia de corriente obtenida del MPPT.

Se demostró la efectividad y robustez del control no lineal en inyectar una corriente sinusoidal a la red eléctrica con factor de potencia muy cercano a la unidad ante cambios bruscos de la irradiancia.

Con el método de control propuesto no existe distorsión en los cruces por cero de la corriente alimentada a la red tal como lo 
manifiesta Li et al. (2012) debido a la conmutación alternada de los transistores del inversor, demostrando la efectividad del controlador en la obtención de una onda de corriente sinusoidal.

Sin embargo, de la desventaja que tienen este tipo de conversores al trabajar en el modo de conducción discontinua debido a la necesidad transistores con altos valores de corriente, esto se ve compensado por la sencillez del control nolineal propuesto y la calidad de energía y eficiencia obtenida.

\section{REFERENCIAS}

Abbou A., Skik N, (2016). Robust Adaptive Integral Backstepping Control for MPPT and UPF of PV System Connected to the Grid. 7th International Renewable Energy Congress (IREC), 1-6. https://doi.org/10.1109/IREC.2016.7478857

Dai M, Marwali M, Jung J, Keyhani A. (2008). A Three-Phase Four-Wire Inverter Control Technique for a Single Distributed Generation Unit in Island Mode. IEEE Transactions on Power Electronics,23(1), 322-331. DOI: 10.1109/TPEL.2007.911816

El Iysaouy L, Baskys A., Bielskis E, Lahbabi M, Oumnad A. (2018). Impact of Flyback Transformer and Switch Parameters on Efficiency of Single Stage Photovoltaic Microinverter. IEEE 2018 Open Conference of Electrical, Electronic and Information Sciences, 1-5. doi:10.1109/eStream.2018.8394122

Ezinwanne O, Zhongwen F, Zhijun L. (2016). Energy Performance and Cost Comparison of MPPT Techniques for Photovoltaics and other Applications. 3rd International Conference on Energy and Environment Research, ICEER, 107, 297-303. DOI: 10.1016/j.egypro.2016.12.156

Fonkwe E, Xiao W, Khadkikar V. (2014). Dynamic Modeling and Control of Interleaved Flyback Module-Integrated Converter for PV Power Applications. IEEE Transactions on Industrial Electronics, 61(3),13771388. DOI: 10.1109/TIE.2013.2258309

Hamad M, Fahmy A, Abdel-Geliel M. (2013). Power Quality Improvement of a Single-Phase Grid- Connected PV System with Fuzzy MPPT Controller. 39th Annual Conference of the IEEE Industrial Electronics, 18391844. DOI: 10.1109/IECON.2013.6699411

Hasan R, Mekhilef S, Seyedmahmoudian M, Horan B. (2017). Grid-connected Insolated PV Microinverters: A Review. Renewable and Sustainable Energy Reviews, 67, 1065-1080. DOI: 10.1016/j.rser.2016.09.082

Islam M, Mekhilef S, Hasan M. (2015). Single Phase Transformerless Inverter Topologies for Grid-Tied Photovoltaic System: A Review. Renewable and Sustainable Energy Reviews, 46. DOI: 10.1016/j.rser.2015.01.009
Ji Y, Jung D, Kim J.G, Kim J.H, Lee T, Won C. (2011). A Real Maximum Power Point Tracking Method for Mismatching Compensation in PV Array Under Partially Shaded Conditions. IEEE Transactions on Power Electronics, 26(4), 1001-1009.

Kareim A, Mansor M. (2013). Efficiency improvement of the maximum power point tracking for PV systems using support vector machine technique. 4th International Conference on Energy and Environment, 16, 1-10. DOI: 10.1088/1755-1315/16/1/012099

Khalil H. (2015). Nonlinear Control. Edinburgh, England: Pearson.

Lee S, Cha W, Kwon B, Kin M. (2016). Discrete-Time Repetitive Control of Flyback CCM Inverter for PV Power Applications, IEEE Transactions on Industrial Electronics,63(2), 976-984. DOI: 10.1109/TIE.2015.2477484

Lee S, Cha W, Kwon J, Kwon B. (2016). Control Strategy of Flyback Microinverter with Hybrid Mode for PV AC Modules. IEEE Transactions on Industrial Electronics, 63(2), 995-1002. DOI: 10.1109/TIE.2015.2481365

Li Y, Oruganti R. (2012). A Low Cost Flyback CCM Inverter for AC Module Application. IEEE Transactions on Power Electronics, 27(3), 1295-1303. DOI: 10.1109/TPEL.2011.2164941

MacAlpine S, Erickson R, Brandemuehl M. (2013). Characterization of Power Optimizer Potential to Increase Energy Capture in Photovoltaic Systems Operating Under Non-Uniform Conditions. IEEE Transactions on Power Electronics, 26(4), 2936 2945. DOI: 10.1109/TPEL.2012.2226476

Macedo G, Martins D, Coelho R. (2016). Design and Comparative Analysis of CL, CLCL and Trap-CL filters for Current Source Inverters, 12th IEEE International Conference on Industry Applications (INDUSON), 1-8.

Romero E, Spagnuolo G, Franquelo L, Ramos C, Suntio T, Xiao W. (2013). Grid Connected Photovoltaic Generations Plants. IEEE Industrial Electronics Magazine, 6(3), 6-20. DOI: 10.1109/MIE.2013.2264540

Zhang Z, He X, Liu Y. (2013). An Optimal Control Method for Photovoltaic Grid-Tied-Interleaved Flyback Microinverters to Achieve High Efficiency in Wide Load Range. IEEE Transactions on Power Electronics, 28(11), $5074 \quad-\quad 5087 . \quad$ DOI: 10.1109/TPEL.2013.2245919 


\section{BIOGRAFÍAS}

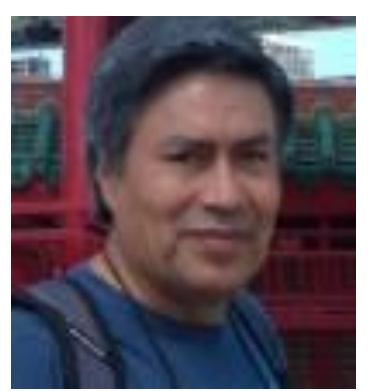

Pablo R. Rivera. Se graduó de Ingeniero en Electrónica y Control en la Escuela Politécnica Nacional, Ecuador en 1986. Obtuvo el grado Master of Science in Power Electronics en la Universidad de Bradford, Inglaterra en 1993, y el grado de $\mathrm{PhD}$. in Electrical Engineering en la Universidad de Louisville, U.S.A. en 2018. Durante su estancia en la Universidad de Louisville, se unió al grupo de Investigación de Sistemas de Energía Eléctrica. Es profesor de la Facultad de Ingeniería y Electrónica de la Politécnica Nacional. Ha dirigido algunos proyectos de grado y de investigación y ha ocupado cargos tales como Coordinador de Carrera, de Postgrado y Decano de la Facultad de Ingeniería Eléctrica y Electrónica. ORCID: https://orcid.org/0000-0002-1228-2063

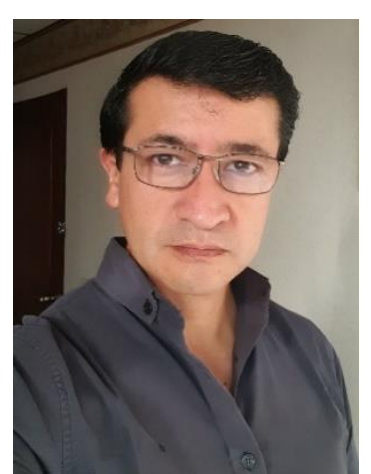

Nelson Gonzalo Sotomayor Orozco, MSc. Nació en QuitoEcuador en septiembre de 1971. Se graduó en la Escuela Politécnica Nacional como Ingeniero en Electrónica y Control en 1999. Obtuvo su título de Magíster en Ingeniería industrial en junio del 2006 en la Escuela Politécnica Nacional. En septiembre del 2008 como becario del Gobierno de México y la Agencia de Cooperación Internacional del Japón (JICA), participó en el IV Curso Internacional de Robótica Aplicada, en el Centro Nacional de Actualización Docente CNAD, CD México. Trabaja como Profesor Principal T/C en el Departamento de Automatización y Control Industrial de la Escuela Politécnica Nacional. Fue Jefe del Departamento de Automatización y Control Industrial (2011- 2013). Desde abril del 2017 se desempeña como Coordinador de la Carrera de Ingeniería en Electrónica y Control. Código ORCID: https://orcid.org/0000-0001-8446-001X 